\title{
A Brief Introduction to Stationary Quantum Chaos in Generic Systems
}

\author{
Marko Robnik* \\ CAMTP - Center for Applied Mathematics and Theoretical Physics, \\ University of Maribor, Mladinska 3, SI-2000 Maribor, SLOVENIA, European Union
}

(Received 26 November, 2019)

\begin{abstract}
We review the basic aspects of quantum chaos (wave chaos) in mixed-type Hamiltonian systems with divided phase space, where regular regions containing the invariant tori coexist with the chaotic regions. The quantum evolution of classically chaotic bound systems does not possess the sensitive dependence on initial conditions, and thus no chaotic behaviour occurs, as the motion is always almost periodic. However, the study of the stationary solutions of the Schrödinger equation in the quantum phase space (Wigner functions or Husimi functions) reveals precise analogy of the structure of the classical phase portrait. In classically integrable regions the spectral (energy) statistics is Poissonian, while in the ergodic chaotic regions the random matrix theory applies. If we have the mixed-type classical phase space, in the semiclassical limit (short wavelength approximation) the spectrum is composed of Poissonian level sequence supported by the regular part of the phase space, and chaotic sequences supported by classically chaotic regions, being statistically independent of each other, as described by the Berry-Robnik distribution. In quantum systems with discrete energy spectrum the Heisenberg time $t_{H}=2 \pi \hbar / \Delta E$, where $\Delta E$ is the mean level spacing (inverse energy level density), is an important time scale. The classical transport time scale $t_{T}$ (transport time) in relation to the Heisenberg time scale $t_{H}$ (their ratio is the parameter $\left.\alpha=t_{H} / t_{T}\right)$ determines the degree of localization of the chaotic eigenstates, whose measure $A$ is based on the information entropy. We show that $A$ is linearly related to the normalized inverse participation ratio. We study the structure of quantum localized chaotic eigenstates (their Wigner and Husimi functions) and the distribution of localization measure $A$. The latter one is well described by the beta distribution, if there are no sticky regions in the classical phase space. Otherwise, they have a complex nonuniversal structure. We show that the localized chaotic states display the fractional power-law repulsion between the nearest energy levels in the sense that the probability density (level spacing distribution) to find successive levels on a distance $S$ goes like $\propto S^{\beta}$ for small $S$, where $0 \leq \beta \leq 1$, and $\beta=1$ corresponds to completely extended states, and $\beta=0$ to the maximally localized states. $\beta$ goes from 0 to 1 when $\alpha$ goes from 0 to $\infty$. $\beta$ is a function of $\langle A\rangle$, as demonstrated in the quantum kicked rotator, the stadium billiard, and a mixed-type billiard.
\end{abstract}

PACS numbers: 01.55.+b, 02.50.Cw, 02.60.Cb, 05.45.Pq, 05.45.Mt

Keywords: quantum chaos, Hamiltonian system, random matrix theory, mixed-type classical phase space, spectral statistics, quantum localization

DOI: https://doi.org/10.33581/1561-4085-2020-23-2-172-191

\section{Introduction and overview}

Quantum chaos is the study of the signatures of classically chaotic behavior in bound Hamiltonian systems [1-3], which have discrete energy spectra. We are interested in the structure and statistical properties of their eigenstates and of their energy spectra. The time evolution of quantum states according to the Schrödinger

\footnotetext{
${ }^{*}$ E-mail: marko.robnik@guest.um.si
}

equation in bound systems is always almost periodic, and there is no sensitive dependence on initial conditions. Any reasonable definition of the asymptotic Lyapunov exponents results in the conclusion that they must vanish. The reason is that the quantum evolution operator (Hamilton operator) in the chaotic regime has discrete spectrum, while in the corresponding classical system the evolution operator (Liouville operator) has continuous spectrum, allowing for chaotic behaviour with positive Lyapunov exponents. Thus the question arises where do we see the 
quantum signatures of classical chaos? It is the structure of the phase space which provides the connection between the classical and quantum picture.

The classical Hamilton systems can have in the general case very complex structure of the invariant components in the $2 N$ dimensional phase space, where $N$ is the number of degrees of freedom. Namely, there can be $N$-dimensional invariant tori on which the almost periodic (or quasiperiodic) motion takes place, there can be chaotic components, and moreover, typically there can be a complex fractal structure of an infinite hierarchy of regular islands surrounded by the chaotic sea, a typical KAM scenario [4]. In the extreme case of ergodicity the entire $(2 N-1)$ dimensional energy surface is a single invariant chaotic component, which can have unstable periodic orbits and cantori, all of which have measure (Liouville volume) zero. The other extreme is the completely integrable system, where the entire phase space is foliated into a set of invariant $N$ dimensional tori, on which the motion is almost (or quasi) periodic. The quantum phase space can be established by introducing the Wigner functions [5] or Husimi functions [6]. If the effective Planck constant $h=2 \pi \hbar$ is sufficiently small, the said functions tend to describe and follow the classical phase space structure up to the given resolution scale determined by $h$.

In the case of the mixed-type classical phase space, we have to distinguish between the quantum regular and irregular (chaotic) eigenstates, an idea proposed qualitatively already in 1973 by Percival [7]. This line of thought lead to the Principle of Uniform Semiclassical Condensation of Wigner functions [8], based on work of Berry [9], Shnirelman [10], Voros [11], and further developed by Veble, Robnik and Liu [12]. In the semiclassical limit $\hbar \rightarrow 0$ the Wigner functions of the eigenstates condense uniformly on the classical invariant component in the classical phase space, and this principle (PUSC) has a great predictive power as demonstrated e.g. in Ref. [12].

The theoretical connection between the classical and quantum structure of the phase space can be established in the semiclassical limit, which is the short wavelength approximation to the exact solution of the stationary (time- independent) Schrödinger equation. The methods are known under the name semiclassical mechanics [1, 2]. There is more about these methods. Namely, the classical dynamics as the ray dynamics of the quantum wave functions is, for example, an analogy of the relationship between the Gaussian ray optics and the wave phenomena of the Maxwell equations describing the electromagnetic field. In general, we can speak of wave chaos rather than quantum chaos, as the analogous phenomena can be found in all wave systems. Nevertheless, unfortunately, the latter more restrictive terminology has been adopted and kept.

The classical dynamics of bounded Hamiltonian systems determines also the statistical properties of the discrete energy spectra of the corresponding eigenstates. For the classically regular motion it predicts Poissonian energy level statistics, while in the classically fully chaotic (ergodic) systems the statistics of Random Matrix Theory (RMT) applies, as conjectured by Bohigas, Giannoni and Schmit [13] in 1984, also by Casati, Valz-Gris and Guarneri [14], proven by Berry [15], Sieber and Richter [16], and by Haake and coworkers [17-20], using the semiclassical techniques based on the Gutzwiller's periodic orbit theory (See Refs. [21-25] and also the books by Stöckmann [1] and Haake [2]).

The intermediate case of the mixed-type Hamilton systems was treated first theoretically by Berry and Robnik [26], and has been analyzed later on in many studies, most accurately by Prosen and Robnik [27]. In this picture the parameter $\mu_{1}$ plays the crucial role, being the relative fraction of the phase space volume occupied by the regular regions in the classical phase space, and it also is the relative density of the regular energy levels in the total quantum spectrum of the underlying system. The spectral statistics for the regular levels is Poissonian.

If there are chaotic regions with the relative volume fractions (and corresponding energy level densities) $\mu_{2}, \mu_{3}, \ldots$, then for each of them the RMT statistics applies. Usually, the dominant chaotic region is by far the largest one, $\mu_{2} \gg$ $\mu_{3}, \ldots$, so that the smaller chaotic regions can be neglected, and we have $\mu_{1}+\mu_{2}=1$.

The best mathematical description of such a mixed-type case is in terms of the gap probability 
$\mathcal{E}(S)$. This is the probability that an energy interval (after unfolding, i.e. reducing the mean energy level density to unity) is empty of levels. Clearly, if regular and chaotic eigenstates are not correlated, being statistically independent of each other, the gap probability simply factorizes, that is

$$
\mathcal{E}(S)=\mathcal{E}_{P}\left(\mu_{1} S\right) \mathcal{E}_{R M T}\left(\mu_{2} S\right),
$$

where $P$ and $R M T$ refer to the Poissonian and RMT statistics, respectively. The Poissonian is $\mathcal{E}_{P}(S)=\exp (-S)$. For the GOE level spacing distribution, which applies if the time reversal symmetry (or any other antiunitary symmetry) exists, the well known Wigner distribution (Wigner surmise) is an excellent analytical approximation

$$
P_{W}(S)=\frac{\pi S}{2} \exp \left(-\frac{\pi S^{2}}{4}\right)
$$

while the corresponding gap probability is

$$
\mathcal{E}_{W}(S)=1-\operatorname{erf}\left(\frac{\sqrt{\pi} S}{2}\right)=\operatorname{erfc}\left(\frac{\sqrt{\pi} S}{2}\right) .
$$

The level spacing distribution $P(S)$ is the second derivative of the gap probability $P(S)=$ $d^{2} \mathcal{E}(S) / d S^{2}$, and therefore in this case given by

$$
\begin{aligned}
P_{B R}(S) & =e^{-\mu_{1} S} e^{-\frac{\pi \mu_{2}^{2} S^{2}}{4}}\left(2 \mu_{1} \mu_{2}+\frac{\pi \mu_{2}^{3} S}{2}\right)(4) \\
& +e^{-\mu_{1} S} \mu_{1}^{2} \operatorname{erfc}\left(\frac{\sqrt{\pi} \mu_{2} S}{2}\right) .
\end{aligned}
$$

as derived by Berry and Robnik [26]. Of course, $\mu_{2}=1-\mu_{1}$. The gap probability $\mathcal{E}(L)$ is just a special case of $\mathcal{E}(k, L)$ probability of finding $k$ levels on an interval of length $L$, namely $\mathcal{E}(L)=\mathcal{E}(0, L)$. For more details about the $\mathcal{E}(k, L)$ probabilities with $k>0$ see Ref. [27].

The above description of generic systems in quantum chaos is correct provided the classical chaos is "fast enough". By this we mean that the classical transport, or diffusion, in the chaotic part of the phase space, in particular in the momentum space, is fast enough. We think of a large ensemble of initial conditions in a small blob, and follow its spreading in time. In systems that are close to integrability, this diffusion can be very slow, mimicking an almost integrable system, and therefore intuitively we expect that also the quantum analogue, at low energies, will behave close to the behavior of an integrable system. An example is the stadium billiard of Bunimovich [28] with small parameter $\epsilon$, that is just slightly distorted circle. Therefore this condition of "fast enough chaos" is crucial for the applicability of the RMT statistics of the corresponding energy spectra, and it also implies that the eigenstates supported by the chaotic components are chaotic, in fact Gaussian random functions [9], and in such case their Wigner functions are entirely extended on the classical invariant chaotic component.

This condition, however, is not always satisfied. The phenomenon of dynamical (or quantum) localization can occur, first discovered and further explored by Chirikov, Izrailev and Shepelyansky [29] in the quantum kicked rotator (QKR) introduced by Casati, Chirikov, Izrailev and Ford [30] as a model system, and later extensively studied in particular by Izrailev [3135]. The QKR is a time periodic (Floquet) system. The time independent chaotic systems are exemplified by the 2-dim billiard systems. Borgonovi, Casati and $\mathrm{Li}$ [36] have studied from this point of view the stadium billiard of Bunimovich [28]. See also the review by Prosen [37]. The case of mixed-type billiard has been studied recently by Batistić and Robnik [38-40].

The criterion for localization is in terms of the ratio

$$
\alpha=\frac{t_{H}}{t_{T}}
$$

of the Heisenberg time $t_{H}$ and the classical transport time $t_{T}$. Here $t_{H}=2 \pi \hbar / \Delta E$, with $\Delta E$ being the mean energy level spacing (inverse energy level density), which is an important time scale in any quantum system with discrete energy spectrum, while $t_{T}$ is the above introduced purely classical ( $\hbar$-independent) diffusion time, or typical time needed for an ensemble of initial sharply distributed momenta to spread uniformly over the classical chaotic component. If $\alpha \ll 1$ the chaotic eigenstates are maximally localized, while if $\alpha \gg 1$ the eigenstates are maximally extended, but in between we have the partially localized eigenstates. The degree of localization can be measured most easily in terms of the Husimi function [6], which is positive definite and can 
be treated as quasi-probability density. There are three main localization measures: $A$, the information entropy measure, $C$ the correlation localization measure, and $n I P R$ the normalized inverse participation ratio. As recently shown $[39,41,42]$, they are all proportional to each other (linearly related) and thus equivalent. The energy spectra of the localized chaotic eigenstates can be well described by the fractional power law level repulsion, $P(S) \propto S^{\beta}$, for small $S$, and $\beta \in[0,1]$ : $\beta=0$ corresponds to the maximal localization and Poissonian statistics, while $\beta=1$ corresponds to the maximal extendedness (delocalization) and the RMT statistics. It has been found that $\beta$ is a function of $A$, they are linearly related in QKR and in the stadium billiard, as well as (approximately) in the mixed-type billiard. It is also an almost rational function of $\alpha$.

The local behaviour of $P(S)$ at small $S$ can be globalized by approximating it by the well known Brody distribution [43, 44], described by the following formula

$$
P_{B}(S)=c S^{\beta} \exp \left(-d S^{\beta+1}\right),
$$

where by normalization of the total probability and the first moment we have

$$
c=(\beta+1) d, \quad d=\left(\Gamma\left(\frac{\beta+2}{\beta+1}\right)\right)^{\beta+1}
$$

with $\Gamma(x)$ being the Gamma function. It interpolates the exponential and Wigner distribution as $\beta$ goes from 0 to 1 . The corresponding gap probability is

$$
\mathcal{E}_{B}(S)=\frac{1}{\gamma(\beta+1)} Q\left(\frac{1}{\beta+1},(\gamma S)^{\beta+1}\right),
$$

where $\gamma=\Gamma\left(\frac{\beta+2}{\beta+1}\right)$ and $Q(a, x)$ is the incomplete Gamma function

$$
Q(a, x)=\int_{x}^{\infty} t^{a-1} e^{-t} d t .
$$

Here the only parameter is $\beta$, the level repulsion exponent in (6), which measures the degree of localization of the chaotic eigenstates: if the localization is maximally strong, the eigenstates practically do not overlap in the phase space (of the Wigner functions) and we find $\beta=0$ and Poissonian distribution, while in the case of maximal extendedness (no localization) we have $\beta=1$, and the RMT statistics of levels applies. Thus, by replacing $\mathcal{E}_{R M T}(S)$ with $\mathcal{E}_{B}(S)$ we get the so-called Berry-Robnik-Brody (BRB) distribution, which generalizes the Berry-Robnik (BR) distribution such that the localization effects are included [38]. In this way the problem of describing the energy level statistics is empirically solved. However, the theoretical derivation of the Brody distribution for the localized chaotic states remains an important open problem.

One important theoretical plausibility argument by Izrailev in support of Brody (or Brody-like) intermediate level spacing distribution is that the joint level distribution of Dyson circular ensembles can be extended to noninteger values of the exponent $\beta$ [35]. The Izrailev distribution is a bit more complicated but has the feature of being a better approximation for the GOE distribution at $\beta=1$. However, recent numerical results show that Brody distribution is slightly better in describing real data $[38,39,45,46]$, and is simpler, which is the reason why we prefer and use it.

There are further important statistical aspects of the quantum localization measures. Namely, it turns out that $A$ has a distribution, described by a probability density. If the classical chaotic region is uniform in the sense of uniform recurrence times, the distribution is universally the beta distribution, as recently shown [41, 42]. On the other hand, if the recurrence times over the chaotic region vary widely, due to the stickiness effects, the distribution becomes nonuniversal, being specific of the classical phase space structure and the dynamical properties. The distribution can have several local maxima, which might be associated with the classical stickiness regions.

In the continuation of this review I shall present an elementary introduction to the quantum chaos of generic systems.

\section{The Schrödinger equation}

Quantum chaos is the study of phenomena in the quantum domain which correspond to the classical chaotic behaviour, in the 
time-independent picture, as explained in the introduction. But let us first consider the solutions of the time-dependent Schrödinger equation of a point particle in the potential $V(\mathbf{q})$,

$$
i \hbar \frac{\partial \psi}{\partial t}=\hat{H} \psi=-\frac{\hbar^{2}}{2 m} \Delta \psi+V(\mathbf{q}) \psi,
$$

where $h=2 \pi \hbar$ is the Planck constant, $\psi(\mathbf{q}, t)$ the wave function depending on the $N$-dim space coordinate vector $\mathbf{q}$ and on time $t, m$ the mass of the point particle moving under the influence of the potential $V(\mathbf{q})$, and $\Delta=\partial^{2} / \partial \mathbf{q}^{2}$ is the $N$-dim Laplace operator. For example, in the case $N=2$ we have

$$
\Delta=\frac{\partial^{2}}{\partial x^{2}}+\frac{\partial^{2}}{\partial y^{2}}
$$

When dealing with specific model systems, especially billiards, we shall restrict ourselves mainly to $N=2$.

We investigate the solutions of the Schrödinger equation and try to relate them to the corresponding classical dynamics, for which obviously the limiting behaviour $\hbar \rightarrow 0$ is of prime interest. The methods and approximations of the semiclassical mechanics offer an important bridge between the classical and quantum mechanics, which is presented in detail in the books $[1,2]$. We also might think of this approximation as short wavelength approximation, which is in general applicable to all wave systems and their solutions.

Furthermore, we shall restrict ourselves to the purely binding potential $V(\mathbf{q})$, such that the classical motion of the particle is bounded (finite) for all initial conditions, and also quantally the particle cannot escape to infinity (no ionization threshold). The energy spectrum of the Hamilton operator $\hat{H}(10)$ is purely discrete and countable infinite. An example of such Hamiltonian system is a classical billiard system, where a point particle is moving freely inside a potential box with hard walls, experiencing an elastic collision when hitting the boundary.

Classically, in Hamiltonian systems, we can have regular, stable, quasiperiodic motion on invariant tori, or irregular, unstable, chaotic motion. The latter one is characterized by the property of the sensitive dependence on initial conditions, which is quantified by the existence of the positive Lyapunov exponents. In this case two nearby orbits in the classical phase space diverge exponentially with time $\propto \exp (\Lambda t)$, and the relevant exponent $\Lambda$ is called the largest positive Lyapunov exponent. In the example of billiard systems, the shape of the boundary determines what kind of dynamics the system exhibits. In quantum mechanics the concept of an orbit and trajectory does not exist due to the Heisenberg uncertainty principle, according to which the product of uncertainties of position $x, \Delta x$, and the corresponding momentum $p_{x}$, $\Delta p_{x}$, satisfy the rigorous inequality $\Delta x \Delta p_{x} \geq$ $\hbar / 2$. This implies that also the divergence of nearby trajectories cannot be defined. Indeed, any attempt to define some kind of meaningful quantum analogue of the asymptotic Lyapunov exponent $\Lambda$ fails in the sense that it is always zero. Therefore, in quantum mechanics the sensitive dependence on initial conditions does not exist, and the time evolution of the wave function $\psi(t)$ as the solution of the time-dependent Schrödinger equation (10) is stable, almost periodic, and reversible: integration of the classical chaotic motion for times much larger than Lyapunov time $\tau=1 / \Lambda$ is fundamentally irreversible once the accuracy of integration is exhausted, while the corresponding quantum evolution of the wave function is still reversible. For details see [2]. Therefore quantum chaos in the sense of positive Lyapunov exponents does not exist. In this sense, in the time evolution the correspondence of the classical and quantum chaos does not exist.

However, as mentioned in the introduction, there is another, stationary, aspect of classical dynamics in Hamiltonian systems, namely the structure of the phase space, the so-called phase portrait, where the phase space is decomposed into the invariant components (regions), each one containing a dense orbit. In the case of classical integrability, to be defined in detail in the next section, all orbits wind quasiperiodically on $N$ dimensional invariant tori $(N$ is the number of the degrees of freedom, that is the dimension of the configuration space), and the entire phase space is foliated into the family of invariant tori. The torus is uniquely labelled by the value of $N$ classical canonical actions $\mathbf{I}$, and the position on the torus is uniquely specified by the $N$ canonically conjugate angles $\theta$. Integrable systems are very special and rare, but important, as we 
can entirely describe them analytically, and also understand what happens (to the phase portrait) if we slightly perturb them, by using a variety of perturbation methods. The opposite extreme is complete chaos, ergodicity, where almost each orbit is chaotic, the set of exceptions having measure zero, and the orbits visit arbitrarily small neighbourhoods of any other point in phase space infinitely many times, as time goes to infinity. Therefore the phase average of functions and the time average are equal. The entire phase space is just one chaotic invariant component. In between there are the mixed-type systems with extremely complex structure of the phase portrait, where regular islands of stability on the invariant tori coexist with chaotic sea surrounding them and which exhibit an infinite hierarchy of statistically selfsimilar structures. It is the fundamental KAM theorem [4] which describes the slightly perturbed Hamiltonian systems. It states that most of the invariant tori survive a perturbation, namely they do still exist after the perturbation with the same $N$ frequencies of the quasiperiodic motion, but are typically slightly distorted. However, the rational tori are destroyed, and in place of them we get an even number of periodic orbits, half of them stable and half of them unstable, surrounded by chaotic region (Poincaré-Birkhoff theorem).

The analogue of the structure of the classical phase portrait in the quantum mechanics is the structure of the eigenfunctions, of their corresponding Wigner functions in the quantum phase space to be defined below, and of the properties of the corresponding energy spectra. As we shall see, in this stationary picture there is a very well defined and rich correspondence between the classical and quantum chaos: The quantum signatures of classical chaos, as the title of Haake's book [2] goes, are very well defined.

To start with, quantally, we have to solve the Schrödinger equation (10) for the eigenstates with sharply defined eigenenergies $E_{n}$, where $\psi(\mathbf{q}, t) \propto \psi_{n}(\mathbf{q}) \exp \left(-i E_{n} t / \hbar\right)$ and the corresponding eigenfunctions $\psi_{n}$ are satisfying the boundary conditions, always requiring the normalizability of $\psi_{n}$, namely $\int\left|\psi_{n}(\mathbf{q})\right|^{2} d^{N} \mathbf{q}<$ $\infty$. In billiards we usually require the Dirichlet boundary conditions, $\psi=0$ on the boundary, but other possibilities, e.g. the Neumann boundary conditions of vanishing normal derivative of $\psi_{n}$, might be interesting, depending on the circumstances.

The eigenstates are defined as the normalizable eigenfunctions of the Hamilton operator $\hat{H}$, namely $\hat{H} \psi_{n}=E_{n} \psi_{n}$. Thus we have to solve the stationary (time-independent) Schrödinger equation,

$$
\frac{\hbar^{2}}{2 m} \Delta \psi_{n}+\left(E_{n}-V(\mathbf{q})\right) \psi_{n}=0 .
$$

In the following sections we shall deal with this task, to characterize different types of solutions $\psi_{n}$ and the associated energy spectra $E_{n}$.

In closing this section let us make it clear that the time-dependent and time-independent Schrödinger equations, (10) and (12), are just special cases of some wave equations. From the mathematical point of view they can be equivalent or similar to some other wave equations of mathematical physics, such as the wave equations describing electromagnetic, acoustic, elastic, seismic waves, water surface waves, etc., where precisely the same questions can be addressed, and the same conclusions can be reached. For details see the books by Stöckmann [1] and Haake [2]. Therefore the terminology "quantum chaos" is much too narrow, and instead, we should speak of "wave chaos". Nevertheless, the name quantum chaos is generally well established, but we should be aware of the wide spectrum of wave phenomena that can occur in almost all wave systems. Of course, understanding the wave chaos is also closely related to the opposite effects of the spontaneous formation of ordered structure in certain wave systems such as e.g. reaction-diffusion systems. It is necessary to understand under what conditions order or chaos can emerge, which is the central question of Haken's fundamental work on synergetics [47].

\section{Quantum mechanics of classically integrable systems}

Integrable Hamiltonian systems are extremely rare but important. The total energy is conserved if their Hamilton function does not depend on time (autonomous system). They are characterized by the existence of invariant $N$-dimensional tori everywhere in the classical phase space. Examples are e.g. 
centrally symmetric potentials where the angular momentum is conserved. In the domain of 2-dimensional billiard systems we have only the rectangle and the elliptic billiard. In the former case the absolute value of the momenta $p_{x}$ and $p_{y}$ are conserved, while in the elliptic billiard [48] the product of the angular momenta with respect to the two foci is the conserved quantity. In the special case of the circle (zero eccentricity) the angular momentum is conserved.

What can we say about such systems? Do their eigenfunctions have some special structure, along with their energy spectra? Let us for a moment concentrate on the 2-dim billiard systems. The general Schrödinger equation, when using appropriate units, reduces to the simple 2dim Helmholtz equation

$$
\frac{\partial^{2} \psi}{\partial x^{2}}+\frac{\partial^{2} \psi}{\partial y^{2}}+E \psi=0
$$

where we have suppressed the index (quantum number(s) ) $n$, and let us assume the Dirichlet boundary conditions $\psi=0$ on the boundary. The answer to the above question is yes. In both billiards the eigenfunctions have an ordered structure, and in both cases the solutions can be easily found, thanks to the separability of the systems. For the rectangle with horizontal width $a$ and the vertical width $b$ it is easy to find the solution given by $\psi_{m, n}(x, y)=C \sin \frac{\pi m x}{a} \sin \frac{\pi n y}{b}$, where the constant $C$ is determined by the normalization. Here $m$ and $n$ are the two quantum numbers (positive integers), associated with and labeling the given eigenfunction. Indeed, the nodal lines defined by the vanishing of $\psi(\mathbf{q})=0$ are the horizontal straight lines $y=j b / n=$ const., where $j=0,1, \ldots, n$, and the vertical lines $x=j a / m=$ const., with $j=0,1, \ldots, m$.

In the circle billiard the nodal lines are defined by the zeros of the radial Bessel functions, which are circles, and by the zeros of the angular trigonometric function, which are polar rays, straight lines, emanating from the center of the circle. If we perturb the two billiards, and solve the Helmholtz equation (13), this structure becomes immediately destroyed by a generic perturbation breaking the separability and integrability of the system. In fully chaotic systems the nodal structure becomes entirely random.
How about the energy spectra $E$ of the integrable billiards, determined by solving (13)? They are characterized by two quantum numbers. In the rectangle billiard with horizontal width $a$ and the vertical width $b$, we find using the above eigenfunctions

$$
E_{m, n}=\pi^{2}\left(\frac{m^{2}}{a^{2}}+\frac{n^{2}}{b^{2}}\right),
$$

where $m, n$ are the two quantum numbers (positive integers). Although this energy spectrum is simple and explicit, its statistical properties are not so simple. As we shall see, the statistical properties of energy spectra are deeply related to the dynamical nature of the underlying Hamiltonian system. If the system is integrable, the statistics is the Poissonian and characteristic of entirely uncorrelated energy levels, while in the case of fully chaotic (ergodic) systems the statistics of the random matrix theory applies. This is to be explained in details in the next section.

Before the statistical analysis is performed, we must prepare the grounds for comparison of quite different systems. In order to achieve that we must unfold the spectrum, which by definition means transformation of the actual energy spectrum $E_{n}$ to the unfolded energy spectrum $e_{n}$, such that the mean spacing $\Delta e=$ $\left\langle\left(e_{n+1}-e_{n}\right)\right\rangle$ of $e_{n}$ is equal to unity everywhere (for all $e$ ). This can be done if we know the mean energy level spacing $\Delta E=1 / \rho(E)$, or the energy level density $\rho(E)$. Then, the unfolding is simply $e_{n}=E_{n} / \Delta E=E_{n} \rho\left(E_{n}\right)$. The density of states is known by the Thomas-Fermi rule of filling the classical phase space inside the energy surface $E=H(\mathbf{q}, \mathbf{p})=$ const . with the Planck cells of volume $(2 \pi \hbar)^{N}$. Namely, the cumulative number $\mathcal{N}$ of the energy eigenvalues below the energy $E$ is given by

$$
\mathcal{N}(E)=\frac{1}{(2 \pi \hbar)^{N}} \int_{H(\mathbf{q}, \mathbf{p}) \leq E} d^{N} \mathbf{q} d^{N} \mathbf{p} .
$$

Therefore,

$$
\rho(E)=\frac{d \mathcal{N}}{d E}=\frac{1}{(2 \pi \hbar)^{N}} \int \delta(E-H(\mathbf{q}, \mathbf{p})) d^{N} \mathbf{q} d^{N} \mathbf{p}
$$

where $\delta(x)$ is the Dirac delta function. For 2-dim billiards with units defined by (13) we can get 
more than that, namely

$$
\mathcal{N}(E)=\frac{\mathcal{A} E}{4 \pi}-\frac{\mathcal{L} \sqrt{E}}{4 \pi}+c . c .
$$

where $\mathcal{A}$ and $\mathcal{L}$ are the area and the circumference of the billiard, while c.c. are some small constants (curvature and corner corrections) which for large $E$ are unimportant. The first term in (17) results directly from (15), while the second one is the socalled perimeter correction. Asymptotically when $E \rightarrow \infty$ the leading term is important and dominant, and is known also as the Weyl formula, as Weyl was the first to derive it.

With the energy spectrum being unfolded we start the statistical analysis. Rather than defining all the correlation functions etc., we shall consider only two statistical measures. The first one is the gap probability $\mathcal{E}(S)$, which is the probability that on an interval of length $S$ there is no level (of the unfolded spectrum). The second one is the level spacing distribution $P(S)$ : The probability to have a level spacing within the interval $(S, S+$ $d S$ ), is equal to $P(S) d S$. It turns out that they are related through $P(S)=d^{2} \mathcal{E}(S) / d S^{2}$ (see e.g. [2]).

For the irrational rectangular billiard ( $a$ and $b$ are not rationally connected) we find the Poissonian statistics, namely

$$
\mathcal{E}(S)=\exp (-S), \quad P(S)=\exp (-S) .
$$

There is no parameter involved in this formula, which is a hint that something similar should be observed in other integrable systems, like the elliptic billiard, of which the circle billiard is a special case. Indeed, this is the case, and we speak of the universality class of the Poissonian spectral statistics of integrable systems. There are some subtleties around this problem, regarding the asymptotic behaviour with increasing energy $E$, which were studied in [49], but the major conclusion about the validity of the Poissonian statistics is confirmed. Intuitively it is easy to understand: If a quantum energy spectrum is characterized by two or more quantum numbers, we will have generically a statistically independent superposition of infinitely many discrete number sequences. Independent of the properties of the individual number sequence, such a superposition always results in a Poissonian sequence.
In the general case of a $N$-dim classically integrable system defined by the Hamilton function $H(\mathbf{q}, \mathbf{p})$ we can perform the construction of such $N$ quantum numbers in terms of the action-angle variables $(\mathbf{I}, \theta)$. In the semiclassical limit of small $\hbar$ the so-called EBK quantization (torus quantization) is based on the quantization of the classical actions $\mathbf{I}$. It is called after Einstein, Brillouin and Keller. The phase space has $2 N$ dimensions, the energy surface $E=H(\mathbf{q}, \mathbf{p})=$ const has $2 N-1$ dimensions, and by definition we have $N$ integrals of motion $A_{j}, j=1, \ldots, N$, of which $A_{1}$ is by convention the Hamilton function, the energy $E=H$. Then, the $N$ dimensional invariant surfaces labelled by $\mathbf{A}$ have the topology of $N$-dimensional tori. The actions - the generalized momenta - are defined by the $N$ irreducible circuit integrals on a torus (such circuits that cannot be continuously shrunk to a point) labeled by $\mathbf{A}$ or $\mathbf{I}$,

$$
I_{j}=\frac{1}{2 \pi} \oint_{C_{j}} \mathbf{p} \cdot d \mathbf{q}
$$

The canonically conjugate angle variables $\theta_{j}$ are defined by using the action integral as a generating function of the underlying canonical transformation. For details see e.g. the references $[4,50]$. The Hamilton function $H(\mathbf{q}, \mathbf{p})$ becomes only a function of $A_{j}$ values, and of $\mathbf{I}$, namely after inverting $A_{j}=A_{j}\left(I_{k}\right)$, it is a function of the actions alone, $A_{1}(\mathbf{q}, \mathbf{p})=H(\mathbf{q}, \mathbf{p})=H(\mathbf{I})$. We say that the angles $\theta_{j}$ are cyclic variables.

For sufficiently small $\hbar$ we obtain the socalled semiclassical torus quantization, or EBK quantization, because we quantize the actions of the tori,

$$
\mathbf{I}_{\mathbf{m}}=\left(\mathbf{m}+\frac{\alpha}{4}\right) \hbar
$$

where $\mathbf{m}$ is a $N$-dim vector of nonnegative integers, and $\alpha=\alpha_{1}, \alpha_{2}, \ldots, \alpha_{N}$ are Maslov indices. The indices $\alpha_{j}, j=1,2, \ldots, N$, count the number of caustics (singularities of the wave function in configuration space) encountered in the configuration space while traversing round the fundamental circuit $C_{j}$. Thus, $\alpha_{j}$ depends on how the invariant torus lies in the phase space and on the structure of its projection singularities in the configuration space. The energy eigenvalues are then equal to the value of the Hamilton function 
at the quantized actions (20),

$$
E_{\mathbf{m}}=H\left(\mathbf{I}_{\mathbf{m}}\right)=H\left(\left(\mathbf{m}+\frac{\alpha}{4}\right) \hbar\right) .
$$

The formula (21) with (20) is basically the higher dimensional generalization of the 1-dim semiclassical quantization, taking into account also the Maslov corrections, which Einstein, Brillouin and Keller were not aware of. It is an approximate solution at small $\hbar$ of the Schrödinger eigenvalue problem (12). For further details see [1].

Now we can explain the origin of the Poissonian statistics for the quantal energy spectra of classically integrable systems: Having $N$ quantum numbers $\mathbf{m}=\left(m_{1}, m_{2}, \ldots, m_{N}\right)$ means generically (in almost all cases, such where no pathological rationalities occur) statistical independent superposition of infinitely many level sequences, and this must result in a Poissonian sequence, unless there are some special and nongeneric rational relationships or correlations between the individual level sequences. For some discussions see e.g. [49], especially regarding the role of rational relationships in rectangle billiard.

\section{Quantum chaos of classically fully chaotic (ergodic) systems}

We now turn to the another extreme case of fully chaotic, ergodic systems. An example of fully chaotic ergodic motion with a positive Lyapunov exponent is the motion of a point particle in the well known stadium billiard, defined as a rectangle with two semicircles on the two opposite sides, as introduced by Bunimovich [28]. Let us assume that the radius of the two semicircles is unity, while the width of the rectangle between them is $\varepsilon$. The billiard system is ergodic and chaotic at any nonzero value of $\varepsilon$, but the typical time for a chaotic trajectory to fill the entire phase space depends strongly on $\varepsilon$. The details of the diffusion-like motion have been recently studied by Lozej and Robnik [51] and its consequences for quantum chaos in reference [52]. If $\varepsilon=1$ this time, called diffusion time, or transport time $t_{T}$, is of order unity, while for very small $\varepsilon$ it becomes very large. Thus, an initial small blob of initial conditions spreads diffusively very quickly in the classical phase space when $\varepsilon=1$, while for small $\varepsilon, t_{T}$ can become very large. As we shall see, this important time scale has to be compared with the important quantum time scale called Heisenberg time (or break time), defined by $t_{H}=$ $2 \pi \hbar / \Delta E$, where $\Delta E$ is the mean energy level spacing of the corresponding quantum system $\Delta E=1 / \rho(E)$ determined by the Thomas-Fermi rule (16). Namely, empirically it is very well observed that the quantum diffusion follows the classical diffusion only up to the Heisenberg time (also called break time), and stops then due to the localization, which is a consequence of the destructive interference effects, and is called dynamical localization or quantum localization, first observed by Casati, Chirikov, Izrailev and Ford in 1979 [30] in the context of time-periodic (Floquet) systems (quantum kicked rotator). If the semiclassical condition is satisfied that the Heisenberg time is larger than all important classical diffusion times in the given system, then we find the universal statistical behaviour of the wave functions and of the energy spectra. Let us recall that according to equation (16) $\Delta E \propto$ $(2 \pi \hbar)^{N}$, and therefore as $\hbar \rightarrow 0$, for $N \geq 2, t_{H}$ will eventually become larger than any $t_{T}$, the latter one being $\hbar$-independent. Thus, at some sufficiently small $\hbar$, in the ultimate semiclassical limit, the quantum localization effects disappear and we can expect the universality to be described in the following.

In contrast to the integrable billiard systems exemplified by the rectangle and the circle, the nodal lines of the stadium billiard with $\varepsilon=$ 1 are entirely irregular, as found already by McDonald and Kaufman [53, 54]. In fact, it has been proposed by Berry [9] that the wave functions of classically fully chaotic billiards is a Gaussian random function, that is the probability amplitude $\psi_{n}(x, y)$ has a Gaussian distribution, and this has been widely confirmed (see e.g. [55]). This indicates that we can expect again some kind of universal behaviour. Regarding the unfolded energy spectrum of this billiard system, it has been shown by Bohigas, Giannoni and Schmit in 1984 [13] that the level spacing distribution $P(S)$ is quite different from the Poissonian, and is approximately but well described by the Wigner distribution (also called Wigner surmise) given by (2) with the corresponding gap probability in (3).

The important feature is the linear behaviour of $P(S)$ at small $S$, starting from 
zero, meaning that the levels tend to be away from a degeneracy, and we speak of level repulsion phenomenon, in this case the linear level repulsion. This must be seen in the contradistinction to the Poissonian $P(S)=e^{-S}$, where the level repulsion is absent and the degeneracies are quite likely. Bohigas, Giannoni and Schmit went on, after numerical exploration of other fully chaotic ergodic billiards with short $t_{T}$, e.g. the Sinai billiard, by proposing what is now known as Bohigas-Giannoni-Schmit (BGS) Conjecture, namely that the statistical properties of the energy spectra of classically fully chaotic and ergodic systems are described by the Random Matrix Theory (RMT), which is one of the fundamental cornerstones of quantum chaos. Some preliminary ideas were published already by Percival [7], and in particular by Casati, Valz-Gris and Guarneri [14].

It has been widely numerically confirmed that the conjecture is correct, and the theoretical foundation has been laid down in the seminal paper by Berry in 1985 [15]. He has shown, using the semiclassical methods developed by Gutzwiller in a series of papers in late 1960's and early 1970's [21-25], that the spectral autocorrelation function and its Fourier transform, the so-called spectral form factor, for small times indeed agree with the RMT. The subject is very difficult, and there was practically no theoretical progress until 2001, when Sieber and Richter [16] extended Berry's work to the next order in power expansion for short times of the form factor. This line of research finally culminated in a series of papers starting in 2006 by Haake and his group [2, 17-20], who succeeded to show that the semiclassical form factor agrees with the RMT to all orders at least up to the Heisenberg time $t_{H}$, and beyond (very recently). Therefore, BGS Conjecture is proven and it is no longer a conjecture but a theorem. The mentioned method of Gutzwiller rests upon the semiclassical calculation of the quantum energy spectral density expressed in terms of a series expansion consisting of contributions stemming from classical periodic orbits. The so-called Gutzwiller trace formula is the stationary phase approximation of the relevant Feynman path integral, used to calculate the Green function. For an excellent introduction see the book by Stöckmann [1].
The RMT has been introduced and developed mainly by Wigner, Dyson, Mehta $[2,56]$ and others to describe statistical properties of the energy spectra of heavy complex nuclei. The main question is what are the statistical properties of the eigenvalues of appropriate ensembles of random matrices, that is matrices with random matrix elements each having a certain probability distribution. It was expected that a large complexity of the physical system results in some randomness. Therefore it was a surprise that it applies also to low-dimensional dynamical systems, such as only two-degrees of freedom billiards, provided that they are fully chaotic (ergodic) and that the semiclassical condition $t_{H}>t_{T}$ is satisfied. The idea is, that if we do not know the details of a complex system, and look at the representation of its Hamilton operator in some basis of the Hilbert space, the matrix elements will appear as random variables.

Due to the lack of space we cannot go into the details of the RMT, and therefore touch only upon the main idea. RMT starts by assuming the Gaussian random distribution of the matrix elements, which are statistically independent of each other, and the distribution is invariant against the transformations that preserve the symmetry of the Hamilton matrices of the ensemble. In the case of real symmetric Hamilton matrices the transformations are orthogonal transformations, and the ensemble of such random matrices is called Gaussian Orthogonal Ensemble (GOE). If $H$ are complex Hermitian matrices, the group of symmetry preserving transformations are the unitary transformations, and the ensemble is called Gaussian Unitary Ensemble (GUE). The central question, among others, is what are the statistical properties of the eigenvalues of such random matrix ensembles.

Rather than going into the full generality, we consider the case of just two-dimensional Gaussian random matrix ensembles, and derive the level spacing distribution for them.

Quite generally, for a Hermitian matrix $\left(\begin{array}{cc}x & y+i z \\ y-i z & -x\end{array}\right)$, with $x, y, z$ real, and $i^{2}=-1$, the two eigenvalues are $\lambda= \pm \sqrt{x^{2}+y^{2}+z^{2}}$ and the level spacing is

$S=\lambda_{1}-\lambda_{2}=2 \sqrt{x^{2}+y^{2}+z^{2}}$. Let us now assume that $x, y, z$ have so far general 
distributions $g_{x}(x), g_{y}(y), g_{z}(z)$, correspondingly. The level spacing distribution is then

$$
\begin{aligned}
P(S) & =\int_{R^{3}} d x d y d z g_{x}(x) g_{y}(y) g_{z}(z) \\
& \times \delta\left(S-2 \sqrt{x^{2}+y^{2}+z^{2}}\right) .
\end{aligned}
$$

The 2D GUE is obtained if we assume $g_{x}(u)=$ $g_{y}(u)=g_{z}(u)=\frac{1}{\sigma \sqrt{\pi}} \exp \left(-\frac{u^{2}}{\sigma^{2}}\right)$, where we perform the necessary normalization $\langle S\rangle=1$, which fixes the $\sigma$. Using the spherical coordinates $r=\sqrt{x^{2}+y^{2}+z^{2}}$ and $\varphi, \theta$, we can perform the integration, followed by the normalization $\langle S\rangle=$ 1, yielding 2D GUE formula

$$
P(S)=\frac{32 S^{2}}{\pi^{2}} \exp \left(-\frac{4 S^{2}}{\pi}\right),
$$

with quadratic level repulsion.

On the other hand, if we restrict the ensemble to the real symmetric class, where we must take $g_{z}(u)=\delta(u)$ while $g_{x}$ and $g_{y}$ remain unchanged Gaussian, and using the polar coordinates $r=\sqrt{x^{2}+y^{2}}$ and $\varphi$, we obtain the 2D GOE formula $P(S)=\frac{\pi S}{2} \exp \left(-\frac{\pi S^{2}}{4}\right)$, with the linear level repulsion, and indeed the result is identical to $P_{W}(S)$ from equation (2).

One should be aware of the fact that there is a clear cut criterion for the GOE or GUE case: If in the system there exists an antiunitary symmetry exemplified by - but not limited to - the time reversal symmetry, then there exists a large and nontrivial basis in the Hilbert space where the representation of the Hamilton operator (matrix) is real symmetric, and GOE statistics applies. If there is no antiunitary symmetry, the system is a general complex Hermitian and the statistics is GUE [56-58].

Note that in both cases of the RMT, like in the Poissonian case of classical integrability, there is no free parameter: Universality. Thus we speak of universality classes of spectral statistics. Hackenbroich and Weidenmüller [59] have shown that the result applies also to a very large class of other random matrix ensembles, provided the limiting distribution of the eigenvalues is smooth and confined to a finite interval, which are quite mild conditions, thus showing that the universality classes are very robust. This has been numerically verified for a number of various non-Gaussian ensembles [60]. One elementary indication for the robustness of the linear level repulsion is demonstrated $[60,61]$ by using (22), assuming $g_{z}(u)=\delta(u)$, and for general $g_{x}, g_{y}$, using the polar coordinates for the integration over the $(x, y)$ plane, we find:

$$
P(S)=\frac{S}{4} \int_{0}^{2 \pi} g_{x}\left(\frac{S}{2} \cos \varphi\right) g_{y}\left(\frac{S}{2} \sin \varphi\right) d \varphi,
$$

and consequently for small $S$ we obtain

$$
P(S)=\frac{\pi S}{2} g_{x}(0) g_{y}(0),
$$

showing, that if $g_{x}, g_{y}$ at $x=0$ and $y=0$ are finite and nonzero, the level repulsion will be always linear, independent of the details of $g_{x}(x), g_{y}(y)$. Indeed, for the Gaussian case, where the normalization $\langle S\rangle=1$ yields $\sigma=1 / \sqrt{\pi}$, we have $g_{x}(0)=g_{y}(0)=1$ and see at once $P(S)=\pi S / 2$ for small $S$, in agreement with (2). The result is easily generalized for the GUE case by using the general $g_{z}(z)$, deriving the quadratic level repulsion, and reproducing (23) for small $S$ in the special case of Gaussian $g_{x}, g_{y}, g_{z}$.

\section{Quantum chaos of classically generic (mixed-type) systems}

In this section we shall consider the mixedtype systems, which are generic, in the sense that almost all Hamilton systems in nature, and also in the mathematical abstract sense, are of this type, the exceptions having measure zero. Namely, classically integrable and fully chaotic (ergodic) systems are exceptional, they have measure zero in the space of Hamilton systems. The generic classical Hamilton systems have divided phase space with regular regions and chaotic systems coexisting. Typically they have extremely complex structure, with infinite self-similar hierarchy of regular islands embedded in chaotic sea, typically having fractal geometry. There are rare exceptions such as the mushroom billiards introduced by Bunimovich, where we have exactly one rigorously integrable (regular) component, and exactly one ergodic chaotic component.

In the general generic case the regular regions consisting of $N$-dim invariant tori coexist 
in the phase space with chaotic regions. The chaotic sea might comprise several disconnected invariant chaotic components. The phase portrait has a very rich structure and is difficult to describe in detail. The quantum mechanics of such systems is also difficult. In the semiclassical limit $\hbar \rightarrow 0$ the quantum mechanics of the stationary Schrödinger equation must somehow tend to the classical mechanics. It was the idea of Percival in 1973 [7] who was the first to suggest, qualitatively, that one should distinguish between the regular eigenstates and the chaotic eigenstates (he called them irregular). However, the question is: How? It is rather obvious that we must introduce some kind of the quantum phase space, where the quantum structures can be compared with the classical ones. This can be achieved by introducing the Wigner functions of the quantum eigenstates.

\section{functions}

5.1. Quantum phase space: The Wigner

The Wigner functions of eigenstates, based on the stationary orthonormal wave functions $\psi_{n}(\mathbf{q})$ in configuration space, are defined in the quantum phase space $(\mathbf{q}, \mathbf{p})$ as follows:

$$
\begin{array}{r}
W_{n}(\mathbf{q}, \mathbf{p})=\frac{1}{(2 \pi \hbar)^{N}} \int d^{N} \mathbf{X} \\
\times \exp \left(-\frac{i}{\hbar} \mathbf{p} \cdot \mathbf{X}\right) \psi_{n}\left(\mathbf{q}-\frac{\mathbf{X}}{2}\right) \psi_{n}^{*}\left(\mathbf{q}+\frac{\mathbf{X}}{2}\right) .
\end{array}
$$

As one can easily see, they are real valued but not positive definite, and possess the following properties:

$(P 1) \quad \int W_{n}(\mathbf{q}, \mathbf{p}) d^{N} \mathbf{p}=\left|\psi_{n}(\mathbf{q})\right|^{2}(=$ probability density in configuration space)

$(P 2) \int W_{n}(\mathbf{q}, \mathbf{p}) d^{N} \mathbf{q}=\left|\phi_{n}(\mathbf{p})\right|^{2}$ (= probability density in momentum space)

$(P 3) \int W_{n}(\mathbf{q}, \mathbf{p}) d^{N} \mathbf{q} d^{N} \mathbf{p}=1$ (normalization)

$(P 4) \quad(2 \pi \hbar)^{N} \int d^{N} \mathbf{q} d^{N} \mathbf{p} W_{n}(\mathbf{q}, \mathbf{p}) W_{m}(\mathbf{q}, \mathbf{p})=$ $\delta_{n m}$ (orthogonality)

$(P 5) \quad\left|W_{n}(\mathbf{q}, \mathbf{p})\right| \leq \frac{1}{(\pi \hbar)^{N}}$ (no singularities; Cauchy-Schwartz inequality)

$(P 6=P 4) \quad \int W_{n}^{2}(\mathbf{q}, \mathbf{p}) d^{N} \mathbf{q} d^{N} \mathbf{p}=\frac{1}{(2 \pi \hbar)^{N}}$ (divergence in the limit $\hbar \rightarrow 0$ )

$(P 7) \quad \hbar \rightarrow 0: \quad W_{n}(\mathbf{q}, \mathbf{p}) \rightarrow(2 \pi \hbar)^{N} W_{n}^{2}(\mathbf{q}, \mathbf{p})>$ 0 (positivity in the limit $\hbar \rightarrow 0$ )
From this one can conclude that in the semiclassical limit $\hbar \rightarrow 0$ the Wigner function becomes predominantly positive definite, that it is supported in a volume cell of size $(2 \pi \hbar)^{N}$, and thus condenses in such a cell, and since the Wigner functions are orthogonal, they must "live" in disjoint supports and therefore become statistically independent of each other. The question is, what is the geometry/structure of such a cell.

5.2. Principle of uniform semiclassical condensation (PUSC) of Wigner functions of eigenstates

The Principle of Uniform Semiclassical Condensation (PUSC) of Wigner functions of eigenstates, based on the papers by Percival [7], Berry [9], Shnirelman [10], Voros [11], Robnik [8], Veble, Robnik and Liu [12], states that the Wigner function $W_{n}(\mathbf{q}, \mathbf{p})$ condenses uniformly on a classical invariant component in the classical phase space, when $\hbar \rightarrow 0$ and if $t_{H}>t_{T}$. This can be an $N$-dim invariant torus, a chaotic component, or the entire energy surface in the case of classical ergodicity:

(C1) Invariant $N$-torus (integrable or KAM):

$$
W_{n}(\mathbf{q}, \mathbf{p})=\frac{1}{(2 \pi)^{N}} \delta\left(\mathbf{I}(\mathbf{q}, \mathbf{p})-\mathbf{I}_{\mathbf{n}}\right) .
$$

(C2) Uniform on topologically transitive (indecomposable invariant) chaotic region:

$$
W_{n}(\mathbf{q}, \mathbf{p})=\frac{\delta\left(E_{n}-H(\mathbf{q}, \mathbf{p})\right) \chi_{\omega}(\mathbf{q}, \mathbf{p})}{\int d^{N} \mathbf{q} d^{N} \mathbf{p} \delta\left(E_{n}-H(\mathbf{q}, \mathbf{p})\right) \chi_{\omega}(\mathbf{q}, \mathbf{p})}
$$

where $\chi_{\omega}(\mathbf{q}, \mathbf{p})$ is the characteristic function on the chaotic component indexed by $\omega$

(C3) Ergodicity (microcanonical Wigner function):

$$
W_{n}(\mathbf{q}, \mathbf{p})=\frac{\delta\left(E_{n}-H(\mathbf{q}, \mathbf{p})\right)}{\int d^{N} \mathbf{q} d^{N} \mathbf{p} \delta\left(E_{n}-H(\mathbf{q}, \mathbf{p})\right.}
$$

Here we also introduce the notation $\mu$ for the relative Liouville measure of the relevant classical 
invariant component indexed by $\omega$ :

$$
\mu(\omega)=\frac{\int d^{N} \mathbf{q} d^{N} \mathbf{p} \delta\left(E_{n}-H(\mathbf{q}, \mathbf{p})\right) \chi_{\omega}(\mathbf{q}, \mathbf{p})}{\int d^{N} \mathbf{q} d^{N} \mathbf{p} \delta\left(E_{n}-H(\mathbf{q}, \mathbf{p})\right)}
$$

This principle turns out to have a great predictive power, as demonstrated and used e.g. in [12]. However, it must be kept in mind that the condition for the uniformity of this semiclassical limit of chaotic states is the fulfillment of the semiclassical condition about the time scales: The Heisenberg time $t_{H}$ must be larger than all relevant classical transport time scales $t_{T}$.

\section{3. $\quad$ Spectral statistics in the mixed-type phase space}

From the PUSC it follows that in the semiclassical limit the eigenstates can be clearly classified as regular and chaotic, just according to the criterion whether they overlap with an invariant $N$-dim torus or with a chaotic region. Using this criterion, one can separate the regular and chaotic eigenstates and perform the statistical analysis separately for each of them. Due to the nonoverlapping supports of the Wigner functions they become statistically independent of each other. Therefore also the corresponding energy level sequences become classified as regular and chaotic, where the regular ones obey the Poissonian statistics and the chaotic ones obey the RMT statistics, provided the semiclassical condition (of time scales) $t_{H}>t_{T}$ is satisfied. Then the total spectrum can be represented as a statistically independent superposition of regular and chaotic level sequences. The regular ones can be lumped together in a single Poissonian sequence, simply because a statistically random superposition of Poissonian level sequences is a Poisson sequence again, while the chaotic sequences must be treated one by one, each of them associated with its relevant supporting classical chaotic region. In such case it becomes obvious that the gap probability $\mathcal{E}(S)$ factorizes: The probability of having no level on interval of length $S$ is the product of probability of having no level of the regular type, times probability of having no level of the chaotic types. In the special case of just one chaotic sequence with the approximate gap probability $\mathcal{E}_{W}(S)=$ $\operatorname{erfc}\left(\frac{\sqrt{\pi} S}{2}\right)$ and one Poissonian sequence with the gap probability $\mathcal{E}_{P}(S)=\exp (-S)$ we obtain

$$
\begin{array}{r}
\mathcal{E}(S)=\mathcal{E}_{P}\left(\mu_{1} S\right) \mathcal{E}_{W}\left(\mu_{2} S\right) \\
=\exp \left(-\mu_{1} S\right) \operatorname{erfc}\left(\frac{\sqrt{\pi} \mu_{2} S}{2}\right) .
\end{array}
$$

Here $\mu_{1}$ is the relative fraction of the phase space volume of the classical regular regions in the classical phase space, while $\mu_{2}=1-\mu_{1}$ is the complementary relative Liouville measure of the chaotic component. Also, $\mu_{1}$ is the mean relative density of the regular energy levels, while $\mu_{2}$ is the mean relative density of the chaotic levels. Since the general relationship $P(S)=$ $d^{2} \mathcal{E}(S) / d S^{2}$ applies, we derive at once the socalled Berry-Robnik level spacing distribution [26] given in Eq. (4). Of course, this probability distribution is normalized $\langle 1\rangle=1$, and has the normalized first moment $\langle S\rangle=1$. It has been tested in many various billiard systems, and in order to see it, since the semiclassical condition (of the time scales) must be satisfied, it is very often necessary to reach the high-lying levels. Most notable confirmation has been achieved by Prosen and Robnik (1994,1999) [27, 62, 63], 1015 years after its derivation. The generalization to many chaotic components is quite straightforward $[8,26]$.

\subsection{Dynamical localization of the chaotic eigenstates}

If the semiclassical condition of time scales $t_{H}>t_{T}$ is not satisfied, the Wigner functions of chaotic eigenstates do not spread out uniformly over the relevant classical chaotic component, but are localized, which means that their effective support is smaller than the classically available chaotic region. For example, this is observed in the stadium billiard if $\varepsilon$ is sufficiently small. It has been shown empirically quite recently [38$40,52]$. that the aspects of dynamical localization in time-independent systems are quite analogous to the dynamical localization phenomena in time-dependent Floquet systems, specifically in quantum kicked rotator [45]. Below we shall show examples of localized chaotic states.

If we neglect the tunneling effects (which couple regular and chaotic levels, breaking the 
statistical independence assumption), which we can do at high energies (small effective $\hbar$, because tunneling effects decrease exponentially with effective $1 / \hbar)$, but treat the dynamical localization effects, we observe empirically [38$40,52,62]$ that the level spacing distribution of the localized chaotic eigenstates is very well captured by the Brody distribution as defined in Eq. (6). Here the only parameter is $\beta$, the level repulsion exponent, which measures the degree of localization of the chaotic eigenstates: if the localization is maximally strong, the eigenstates practically do not overlap in the phase space (of the Wigner functions) and we find $\beta=0$ and Poissonian distribution, while in the case of maximal extendedness (no localization) we have $\beta=1$, and the RMT statistics of levels applies. Thus, by replacing $\mathcal{E}_{W}(S)$ with $\mathcal{E}_{B}(S)$ we get the BRB (Berry-Robnik-Brody) distribution, which generalizes the Berry-Robnik distribution such that the localization effects are included [38].

\section{The billiard systems and Poincaré-Husimi functions}

Having established the formalism of Wigner functions as a kind of the quantum phase space, we now wish to actually look at the Wigner functions in mixed-type quantum systems, in order to separate the regular and the chaotic ones, by simply looking at whether the given eigenstate $W_{n}(\mathbf{q}, \mathbf{p})$ overlaps with a classical regular or classical chaotic region. Furthermore, the question arises whether the chaotic Wigner function is localized or extended on the classical chaotic component. The method and approach is general, but technically difficult to implement in general. Therefore we have to choose some representative model system. The billiard systems are most suitable for such studies.

For a $2 \mathrm{D}$ billiard the most natural coordinates in the phase space $(s, p)$ are the arclength $s$ round the billiard boundary, $s \in$ $[0, \mathcal{L}]$, where $\mathcal{L}$ is the circumference, and the sine of the reflection angle, which is the component of the unit velocity vector tangent to the boundary at the collision point, equal to $p$, which is the canonically conjugate momentum to $s$. These are the Poincaré-Birkhoff coordinates. The bounce map $\left(s_{1}, p_{1}\right) \rightarrow\left(s_{2}, p_{2}\right)$ is area preserving [48], and the phase portrait does not depend on the speed (or energy) of the particle. Quantum mechanically we have to solve the stationary Schrödinger equation, which in a billiard is just the Helmholtz equation $\Delta \psi+k^{2} \psi=0$ with the Dirichlet boundary conditions $\left.\psi\right|_{\partial \mathcal{B}}=0$. The energy is $E=k^{2}$. The important quantity is the boundary function

$$
u(s)=\mathbf{n} \cdot \nabla_{\mathbf{r}} \psi(\mathbf{r}(s)),
$$

which is the normal derivative of the wavefunction $\psi$ at the point $s$ ( $\mathbf{n}$ is the outward unit normal vector). It satisfies the integral equation

$$
u(s)=-2 \oint d t u(t) \mathbf{n} \cdot \nabla_{\mathbf{r}} G(\mathbf{r}, \mathbf{r}(t)),
$$

where $G\left(\mathbf{r}, \mathbf{r}^{\prime}\right)=-\frac{i}{4} H_{0}^{(1)}\left(k\left|\mathbf{r}-\mathbf{r}^{\prime}\right|\right)$ is the Green function in terms of the Hankel function $H_{0}^{(1)}(x)$. It is important to realize that boundary function $u(s)$ contains complete information about the wavefunction at any point $\mathbf{q}$ inside the billiard by the equation

$$
\psi_{j}(\mathbf{r})=-\oint d t u_{j}(t) G(\mathbf{r}, \mathbf{r}(t)) .
$$

Now we go over to the quantum phase space. We can calculate the Wigner functions based on $\psi_{n}(\mathbf{r})$ and perform the procedure developed in the previous section. However, in billiards it is advantageous to calculate the Poincaré-Husimi functions. The Husimi functions are generally just Gaussian smoothed Wigner functions. Such smoothing makes them positive definite, so that we can treat them somehow as quasi-probability densities in the quantum phase space, and at the same time we eliminate the small oscillations of the Wigner functions around the zero level, which do not carry any significant physical contents, but just obscure the picture. Thus, following Tualle and Voros [64] and Bäcker et al [65], we introduce [39] the properly $\mathcal{L}$-periodized coherent states centered at $(q, p)$, as follows

$$
\begin{aligned}
c_{(q, p), k}(s) & =\sum_{m \in \mathbf{Z}} \exp \{i k p(s-q+m \mathcal{L})\}( \\
& \times \exp \left(-\frac{k}{2}(s-q+m \mathcal{L})^{2}\right) .
\end{aligned}
$$


The Poincaré-Husimi function is then defined as the absolute square of the projection of the boundary function $u(s)$ onto the coherent state, namely

$$
H_{j}(q, p)=\left|\int_{\partial \mathcal{B}} c_{(q, p), k_{j}}(s) u_{j}(s) d s\right|^{2} .
$$

In figure 1 we show examples of a regular and of a chaotic eigenstate for the billiard introduced in $[66,67]$ with $\lambda=0.15$.

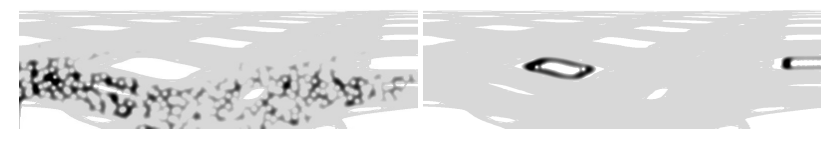

FIG. 1. Examples of a chaotic (left) and a regular (right) state in the Poincaré-Husimi representation. $k_{j}(M)$ are: chaotic: $k_{j}(M)=2000.0181794(0.981)$; regular: $k_{j}(M)=2000.0777155(-0.821)$. The gray background is the classically chaotic invariant component. We show only one quarter of the surface of section $(s, p) \in[0, \mathcal{L} / 2] \times[0,1]$, because due to the reflection symmetry and time-reversal symmetry the four quadrants are equivalent.

Now the classification of eigenstates can be performed by their projection onto the classical surface of section. As we are very deep in the semiclassical regime we do expect with probability one that either an eigenstate is regular or chaotic, with exceptions having measure zero, ideally. To automate this task we have ascribed to each point on the grid a number $A_{i, j}$ whose value is either +1 if the grid point lies within the classical chaotic region or -1 if it belongs to a classical regular region. Technically, this has been done as follows. We have taken an initial condition in the chaotic region, and iterated it up to about $10^{10}$ collisions, enough for the convergence (within certain very small distance). Each visited cell $(i, j)$ on the grid has then been assigned value $A_{i, j}=+1$, the remaining ones were assigned the value -1 .

The Poincaré Husimi function $H(q, p)(36)$ (normalized) was calculated on the grid points and the overlap index $M$ was calculated according to the definition $M=\sum_{i, j} H_{i, j} A_{i, j}$. In practice, $M$ is not exactly +1 or -1 , but can have a value in between. There are two reasons: the finite discretization of the phase space (the finite size grid), and the finite wavelength (not sufficiently small effective Planck constant, for which we can take just $\left.1 / k_{j}\right)$. If so, the question is, where to cut the distribution of the $M$-values, at the threshold value $M_{t}$, such that all states with $M<M_{t}$ are declared regular and those with $M>M_{t}$ chaotic.

There are two natural criteria: (I) The classical criterion: the threshold value $M_{t}$ is chosen such that we have exactly $\mu_{1}$ fraction of regular levels and $\mu_{2}=1-\mu_{1}$ of chaotic levels. (II) The quantum criterion: we choose $M_{t}$ such that we get the best possible agreement of the chaotic level spacing distribution with the Brody distribution (6), which is expected to capture the dynamical localization effects of the chaotic levels.

Let us now separate the regular and chaotic eigenstates and the corresponding eigenvalues, after unfolding, according to our method, using the classical criterion (I). The corresponding threshold value of the index $M$ is found to be $M_{t}=0.431$. The level spacing distributions are shown in figure 2 . As we see, we have perfect Brody distribution with $\beta=0.444$ for the chaotic levels and almost pure Poisson for the regular levels.
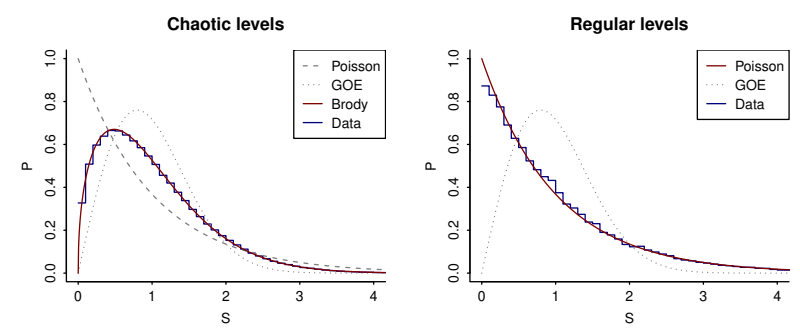

FIG. 2. (color online) Separation of levels using the classical criterion $M_{t}=0.431$. (Left:) The level spacing distribution for the chaotic subspectrum, after unfolding, in perfect agreement with the Brody distribution $\beta=0.444$. (Right:) The level spacing distribution for the regular part of the spectrum, after unfolding, in excellent agreement with Poisson.

\section{The localization measures: A, C} and nIPR

After separating the regular and chaotic eigenstates we want to quantify the degree of localization of the chaotic eigenstates [40]. We express the localization 
measures in terms of the discretized Husimi function. For the entropy localization measure denoted by $A$ we write $A=e^{I} / N_{c}$, where $I=-\int d q d p H(q, p) \ln \left((2 \pi \hbar)^{f} H(q, p)\right)$ is the information entropy and $N_{c}$ is a number of cells on the classical chaotic domain. As we expect fluctuations of $A$ even in the case of full (ergodic) chaos, due to the scars [68] and regions of stickiness $[69,70]$, we have to average $A$ over some set of consecutive chaotic eigenstates. In the case of uniform distribution $H_{i j}=1 / N_{C}$ the localization measure is $A=1$, while in the case of the strongest localization $I=0$, and $A=1 / N_{C} \approx 0$.

For the correlation localization measure denoted by $C$, we first calculate the overlap (correlation matrix) $C_{n m}=\frac{1}{Q_{n} Q_{m}} \sum_{i j} H_{i j}^{n} H_{i j}^{m}$, where $Q_{n}=\sqrt{\sum_{i j}\left(H_{i j}^{n}\right)^{2}}$ is the normalizing factor. Then $C=\left\langle C_{n m}\right\rangle$, and the averaging is over all $n, m$ and a large number of consecutive chaotic eigenstates.

Again we use the billiard like in section 6 with $\lambda=0.15$. For a good approximation of the localization measures $A$ and $C$ it was sufficient to separate and extract about 1.500 consecutive chaotic eigenstates. The two localization measures are linearly equivalent as shown in figure 3 . To get a good estimate of $\beta$ we need much
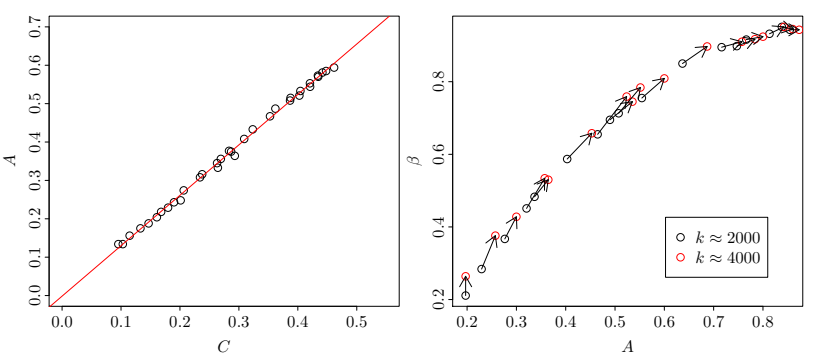

FIG. 3. (color online) (Left:) Linear relation between the two entirely different localization measures, namely the entropy measure $A$ and the correlation measure $C$, calculated for several different billiards at $k \approx 2000$ and $k \approx 4000$. (Right:) We show the functional relation between $\beta$ and the localization measure $A$. Arrows connect points corresponding to the same $\lambda$ at two different $k$.

more levels, and the separation of eigenstates is then technically too demanding. We have instead calculated spectra on small intervals around $k \approx 2000$ and $k \approx 4000$, about 100.000 consecutive levels (no separation), and obtained $\beta$ by fitting the $P(S)$ by the BRB distribution derived in section (5.4) using the classical $\mu_{1}$. The dependence of $\beta$ on $A$ is shown in figure 3. For aesthetic reasons we have rescaled the measure $A \rightarrow A / A_{\max }$ such that it goes from 0 to 1 . The maximal value of $A=A_{\max }=0.68$ was found over 1500 consecutive states of the almost fully chaotic $\lambda=0.25$ billiard. Thus for fully chaotic systems the procedure always yields $A=$ 1. Namely, in real chaotic eigenstates we never reach a perfectly uniform distribution $H(q, p)$, since they always have some oscillatory structure.

We clearly see that there is a functional relationship between $A$ and $\beta$. By increasing $k$ from 2000 to 4000 we increase the dimensionless parameter $\alpha$ by factor 2, therefore $A$ must increase, but precisely in such a way, that the empirical points stay on the scaling curve, as it is observed and indicated by the arrows. We do not have yet a semiempirical functional description of the relationship $\beta(A)$ we found in figure 3 . In the quantum kicked rotator it is just almost linear $[35,45,46]$. Similarly it is found to be almost linear in the stadium of Bunimovich, as recently published in reference [52] and shown in figure 4. Also, $\beta$ is found to be a unique function of

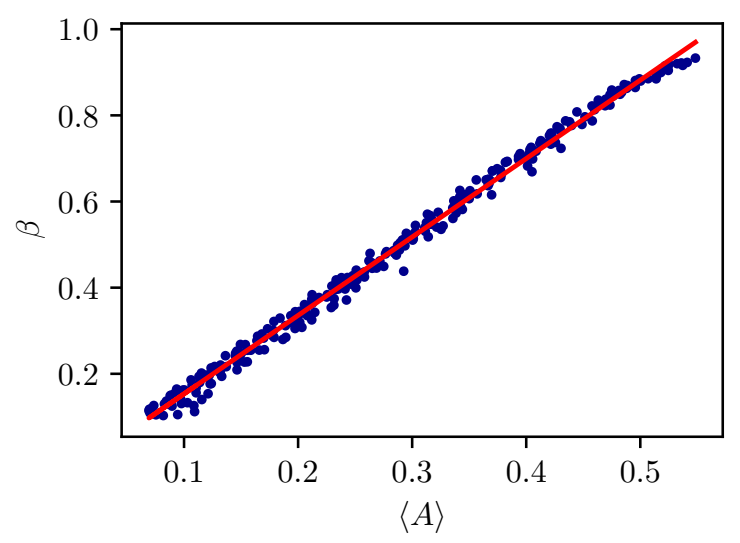

FIG. 4. (color online) The level repulsion exponent $\beta$ as a function of the entropy localization measure $A$ for variety of stadia of different shapes $\varepsilon$ and energies $E=k^{2}$.

$\alpha=t_{H} / t_{T}$, well described empirically by the rational function

$$
\beta=\beta_{\infty} \frac{s \alpha}{1+s \alpha}
$$




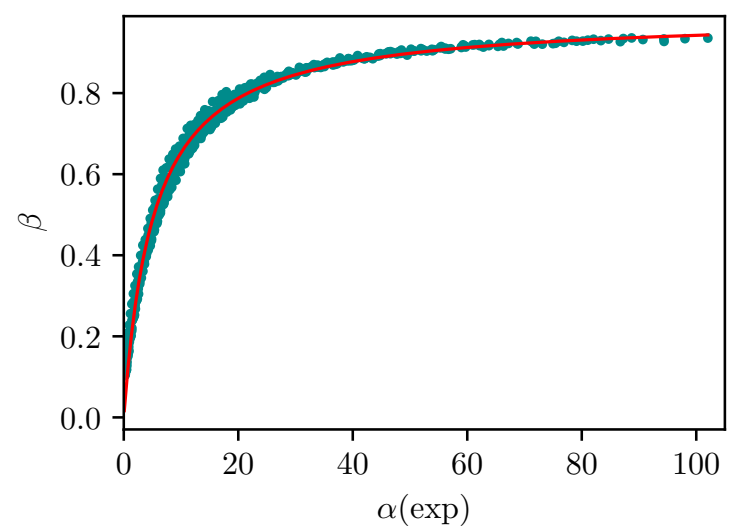

FIG. 5. (color online) The level repulsion exponent $\beta$ as a function of $\alpha$ fitted by the function (37), based on $t_{T}$ from the exponential diffusion law, for variety of stadia of different shapes $\varepsilon$ and energies $E=k^{2}$, as in figure 4 . Here $\beta_{\infty}=0.98$ and $s=0.20$.

as seen in figure 5. For details see reference [52]. Nevertheless, one should observe some scattering of points around the mean value, noted already by Izrailev [35] in the case of the quantum kicked rotator, which probably is due to the fact that the localization measure has a certain distribution rather than a sharp value, as has been observed recently in the kicked rotator by Manos and Robnik [71].

Indeed, in a very recent work [42] we have improved the statistical significance of the data $\beta$ and $A$, by analyzing many different values of $\lambda$, where the relationship $\beta(A)$ is now closer to the linear law than in Fig. 3, where only $\lambda=0.15$ was used. Moreover, we have studied the statistical properties of $A$. It is found that each distribution in the fully chaotic case without stickiness regions can be very well characterized and described by the so-called beta distribution

$$
P(A)=C A^{a}\left(A_{0}-A\right)^{b},
$$

where $A_{0}$ is the upper limit of the interval $\left[0, A_{0}\right]$ on which $P(A)$ is defined, and the two exponents $a$ and $b$ are positive real numbers, while $C$ is the normalization constant such that $\int_{0}^{A_{0}} P(A) d A=$ 1 , i.e.

$$
C^{-1}=A_{0}^{a+b+1} B(a+1, b+1),
$$

where $B(x, y)=\int_{0}^{1} t^{x-1}(1-t)^{y-1} d t$ is the beta function. We shall also use the cumulative distribution defined as

$$
W(A)=\int_{0}^{A} P(x) d x .
$$

Thus we have

$$
\langle A\rangle=A_{0} \frac{a+1}{a+b+3},
$$

and for the second moment

$$
\left\langle A^{2}\right\rangle=A_{0}^{2} \frac{(a+2)(a+1)}{(a+b+4)(a+b+3)}
$$

and therefore for the standard deviation $\sigma$,

$$
\sigma^{2}=A_{0}^{2} \frac{(a+2)(b+2)}{(a+b+4)(a+b+3)^{2}},
$$

such that asymptotically $\sigma \approx A_{0} \frac{\sqrt{b+2}}{a}$ when $a \rightarrow \infty$. Whenever we compare $A$ from different $\lambda$, we have divided $A$ by the relative fraction of the chaotic component in the classical phase space, denoted by $\chi_{C}$, as computed and listed in the table of Ref. [69]. In all cases for $A_{0}$ we have chosen the empirically best value $A_{0}=0.7$, compatible with $A_{\max }=0.68$ estimated before. However, if the classical phase space possesses regions of stickiness, the distribution $P(A)$ becomes nouniversal, specific of the dynamical system and can have quite complex structure with several local maxima, which presumably can be associated and supported by the stickiness regions. For further details see Ref. [69].

As mentioned before, the definition of localization measures can be diverse, and the question arises to what extent are the results objective and possibly independent of the definition. Indeed, as shown, $A$ (information entropy measure) and $C$ (based on the corelations) are linearly related and thus equivalent. Moreover, we have introduced also the normalized inverse participation ratio $n I P R$, defined as follows

$$
n I P R=\frac{1}{N} \frac{1}{\sum_{i, j} H_{i j}^{2}},
$$

for each individual eigenstate $m$. Here $N$ is the number of all grid points, in our specific case $N=400 \times 400=160.000$. However, because we expect fluctuations of the localization measures 
even in the quantum ergodic regime (due to the scars etc), we must perform some averaging over an ensemble of eigenstates, and for this we have chosen 100 consecutive eigenstates. Then, by doing this for all possible data for the billiard at various $\lambda$ and $k$, we ended up with the result that the $n I P R$ and $A$ are linearly related and thus also equivalent, as shown in Fig. 6. This is in perfect agreement with the most recent results for the stadium billiard [41], and thus we believe that it is generally true, independent of a specific model system. It is therefore highly satisfactory that the three entirely different localization measures are equivalent. For details see Refs. [41, 42]. Finally, there is a great lack in

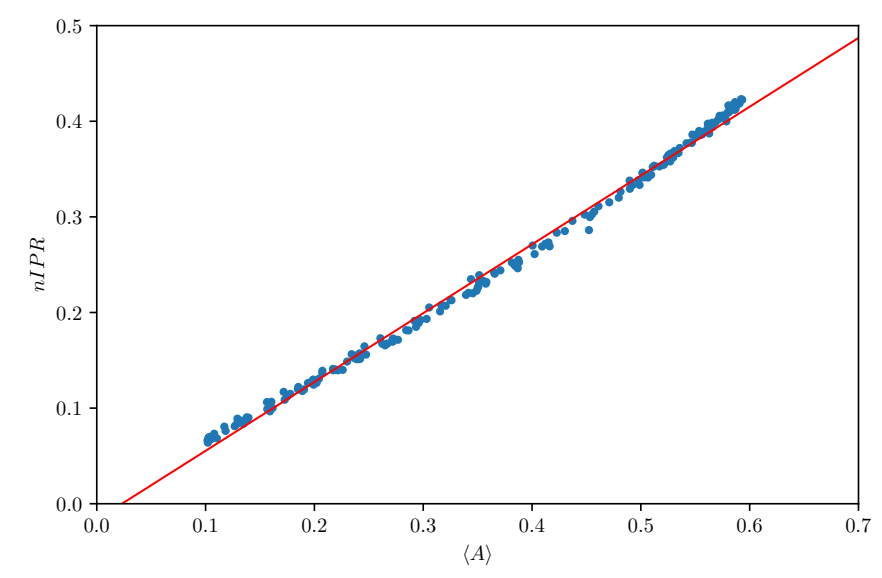

FIG. 6. (color online) The normalized inverse participation ratio as a localization measure, as a function of $A$. They are linearly related and thus equivalent. We have used the chaotic states with $M \geq$ $M_{t}=0.5$. The slope is 0.72 and the intercept -0.017 .

theoretical understanding of the physical origin of the relationship $\beta(A)$, even in the case of (the long standing research on) the quantum kicked rotator, except for the intuitive idea, that energy spectral properties should be only a function of the degree of localization, because the localization gradually decouples the energy eigenstates and levels, switching the linear level repulsion $\beta=1$ (extendedness) to a power law level repulsion with exponent $\beta<1$ (localization). The full physical explanation is open for the future.

\section{Conclusions and discussion}

Quantum chaos, or wave chaos, is a study of the signatures of classical chaos of the underlying classical dynamical system, or more generally, of the ray dynamics of the limiting short-wavelength asymptotics. The time evolution of the bound quantum systems (with purely discrete spectrum) is always almost periodic, stable and reversible in the sense of the absence of the sensitive dependence on initial conditions, and thus it is quite different from the classically chaotic behaviour exhibiting sensitive dependence on initial conditions, due to the positive Lyapunov exponents. In this sense there is no quantum chaos. However, in the time-independent domain, when we look at the eigenstates of the stationary Schrödinger equation, we uncover a complexity of the solutions, fully revealed in the quantum phase space of the Wigner functions, which exactly correspond to the structure of the classical phase space. In the semiclassical limit of sufficiently small effective Planck constant the Heisenberg time is larger than all classical transport (diffusion) time scales, and the chaotic eigenstates (their Wigner functions) are uniformly extended over the entire available chaotic region. Then the regular eigenstates "live" on invariant tori, while the chaotic ones are associated with the chaotic components. The energy spectra belong to the universality classes as for their statistical properties: the regular spectra obey Poissonian statistics, while the chaotic ones obey the statistics of the Gaussian random matrix theory. If the semiclassical condition on the time scales is not satisfied, the chaotic Wigner functions are localized due to the quantum (or dynamical) localization. The degree of localization (localization measures) can be defined in various ways, but different localization measures are found to be equivalent (linearly related). The degree of localization uniquely determines the Brody spectral parameter which enters in the level spacing distribution of localized chaotic eigenstates. Using the overlap criterion for the Wigner functions (or Husimi functions) one can separate the regular and chaotic states, and perform the statistical analysis separately. It is confirmed, in the sense of Percival (1973) and Berry and Robnik (1984), as generalized by Batistić and Robnik (2010-2013), that the 
regular levels are Poissonian, while the localized chaotic ones are Brody-like, where the Brody parameter is a unique function of the localization measure. When we go from the semiclassical limit to larger values of the effective Planck constant (in billiards it means to lower energies), we reveal the tunneling (correlation) effects, where the regular and chaotic eigenstates start to overlap considerably, and thus no longer can be classified clearly as regular or chaotic. The picture becomes quite complex, and is subject of current research.

While the fully chaotic and regular eigenstates are generally very well understood, the description of the localized chaotic states, and of the mixed-type systems, is still open for further investigation. In particular, we need to derive a theory of dynamical localization of stationary chaotic eigenstates, including the derivation of the phenomenological Brody level spacing distribution of such localized chaotic eigenstates. Thus, quite a few fundamental questions of quantum chaos are open for the future.

\section{Acknowledgement}

This work was supported by the Slovenian Research Agency (ARRS) under the grant J19112.

\section{References}

[1] H.-J. Stöckmann, Quantum Chaos - An Introduction (Cambridge University Press, Cambridge, 1999).

[2] F. Haake, Quantum Signatures of Chaos (Springer, Berlin, 2001).

[3] M. Robnik, Eur. Phys. J. Special Topics 225, 959 (2016).

[4] E. Ott, Chaos in Dynamical Systems (Cambridge, Cambridge University Press, 1993).

[5] E. Wigner, Phys. Rev. 40, 749 (1932).

[6] K. Husimi, Proc. Phys. Math. Soc. Jpn. 22, 264 (1940).

[7] I. C. Percival, J. Phys B: At. Mol. Phys. 6, L229 (1973).

[8] M. Robnik, Int. J. Nonlinear Phen. in Compl. Syst. 1, 1 (1998).

[9] M. V. Berry, J. Phys. A: Math. Gen. 10, 2083 (1977).

[10] B. Shnirelman, Uspekhi Matem. Nauk 29, 181 (1974).

[11] A. Voros, Lect. Notes Phys. 93, 326 (1979).

[12] G. Veble, M. Robnik, and J. Liu, J. Phys. A: Math. Theor. 32, 6423 (1999).

[13] O. Bohigas, M. J. Giannoni, and C. Schmit, Phys. Rev. Lett. 52, 1 (1984).

[14] G. Casati, F. Valz-Gris, and I. Guarneri, Lett. Nuovo Cimento 28, 279 (1980).

[15] M. V. Berry, Proc. Roy. Soc. Lond. A 400, 229 (1985).

[16] M. Sieber and K. Richter, Phys. Scr. T90, 128 (2001).
[17] S. Müller, S. Heusler, P. Braun, F. Haake, and A. Altland, Phys. Rev. Lett. 93, 014103 (2004).

[18] S. Heusler, S. Müller, P. Braun, and F. Haake, J. Phys.A: Math. Gen. 37, L31 (2004).

[19] S. Müller, S. Heusler, P. Braun, F. Haake, and A. Altland, Phys. Rev. E 72, 046207 (2005).

[20] S. Müller, S. Heusler, A. Altland, P. Braun, and F. Haake, New J. of Phys. 11, 103025 (2009).

[21] M. C. Gutzwiller, J. Math. Phys. 8, 1979 (1967).

[22] M. C. Gutzwiller, J. Math. Phys. 10, 1004 (1969)

[23] M. C. Gutzwiller, J. Math. Phys. 11, 1791 (1970).

[24] M. C. Gutzwiller, J. Math. Phys. 12, 1791 (1971).

[25] M. C. Gutzwiller, Phys. Rev. Lett. 45, 150 (1980).

[26] M. V. Berry and M. Robnik, J. Phys. A: Math. Gen. 17, 2413 (1984).

[27] T. Prosen and M. Robnik, J. Phys. A: Math. Gen. 32, 1863 (1999).

[28] L. Bunimovich, Commun. Math. Phys. 65, 295 (1979).

[29] B. V. Chirikov, F. M. Izrailev, and D. L. Shepelyansky, Sov. Sci. Rev. C 2, 209 (1981).

[30] G. Casati, B. V. Chirikov, F. M. Izrailev, and J. Ford, Lecture Notes in Physics 93, 334 (1979).

[31] F. M. Izrailev, Phys. Rev. Lett. 56, 541 (1986).

[32] F. M. Izrailev, Phys. Lett. A 125, 250 (1987).

$33]$ F. M. Izrailev, Phys. Lett. A 134, 13 (1988).

[34] F. M. Izrailev, J. Phys. A: Math. Gen. 22, 865 (1989). 
[35] F. M. Izrailev, Phys. Rep. 196, 299 (1990).

[36] F. Borgonovi, G. Casati, and B. Li, Phys. Rev. Lett. 77, 4744 (1996).

[37] T. Prosen, in Proc. of the Int. School in Phys. "Enrico Fermi Course CXLIII, Eds. G. Casati and U. Smilansky (Amsterdam: IOS Press, 2000).

[38] B. Batistić and M. Robnik, J. Phys. A: Math. Theor. 43, 215101 (2010).

[39] B. Batistić and M. Robnik, Phys. Rev. E 88, 052913 (2013).

[40] B. Batistić and M. Robnik, J. Phys. A: Math. Theor. 46, 315102 (2013).

[41] B. Batistić, Č. Lozej, and M. Robnik, Int. J. Nonlinear Phen. in Compl. Syst. 23, 17 (2020).

[42] B. Batistić, Č. Lozej, and M. Robnik, Phys. Rev. E 100, 062208 (2019).

[43] T. A. Brody, Lett. Nuovo Cimento 7, 482 (1973).

[44] T. A. Brody, J. Flores, J. B. French, P. A. Mello, A. Pandey, and S. S. M. Wong, Rev. Mod. Phys. 53, 385 (1981).

[45] T. Manos and M. Robnik, Phys. Rev. E 87, 062905 (2013).

[46] B. Batistić, T. Manos, and M. Robnik, EPL 102, 50008 (2013).

[47] H. Haken, Synergetics (Berlin, Springer, 2004).

[48] M. V. Berry, Eur. J. Phys. 2, 91 (1981).

[49] M. Robnik and G. Veble, J. Phys. A: Math. Theor. 31, 4669 (1998).

[50] V. I. Arnold, Mathematical Methods of Classical Mechanics (New York, Springer, 1980).

[51] Č. Lozej and M. Robnik, Phys. Rev. E 97, 012206 (2018).

[52] B. Batistić, Č. Lozej, and M. Robnik, Int. J. Nonlinear Phenomena in Complex Systems 21, 225 (2018).
[53] S. W. McDonald and A. N. Kaufman, Phys. Rev. Lett. 42, 1189 (1979).

[54] S. W. McDonald and A. N. Kaufman, Phys. Rev. A 37, 3067 (1979)

[55] B. Li and M. Robnik, J. Phys. A: Math. Gen. 27, 5509 (1994).

[56] M. L. Mehta, Random Matrices (Academic Press, Boston, 1991).

[57] M. Robnik and M. V. Berry, J. Phys. A: Math. Gen. 19, 669 (1986).

[58] M. Robnik, Lect. Notes Phys. 263, 120 (1986).

[59] G. Hackenbroich and H. Weidenmüller, Phys. Rev. Lett. 74, 4118 (1995).

[60] M. Robnik, H. M. David, G. Vidmar, and V. G. Romanovski, Int. J. Nonlinear Phen. in Compl. Syst. 13, 13 (2010).

[61] S. Grossmann and M. Robnik, Z. Naturforschung A 62, 471 (2007).

[62] T. Prosen and M. Robnik, J. Phys. A: Math. Gen. 27, L459 (1994).

[63] T. Prosen and M. Robnik, J. Phys. A: Math. Gen. 27, 8059 (1994).

[64] J. Tualle and A. Voros, Chaos Solitons Fractals 5, 1085 (1995).

[65] A. Bäcker, S. Fürstberger, and R. Schubert, Phys. Rev. E 70, 036204 (2004).

[66] M. Robnik, J. Phys. A: Math. Gen. 16, 3971 (1983).

[67] M. Robnik, J. Phys. A: Math. Gen. 17, 1049 (1984).

[68] E. J. Heller, Phys. Rev. Lett. 53, 1515 (1984).

[69] Č. Lozej and M. Robnik, Phys. Rev. E 98, 022220 (2018).

[70] Č. Lozej, Phys. Rev. E 101, 052204 (2019).

[71] T. Manos and M. Robnik, Phys. Rev. E 91, 042904 (2015). 